Textures and Microstructures, Vol. 33, pp. 139-149 Reprints available directly from the publisher Photocopying permitted by license only
(C) 1999 OPA (Overseas Publishers Association) N.V.

Published by license under the Gordon and Breach Science Publishers imprint. Printed in Malaysia.

\title{
TEXTURE ANALYSIS OF QUARTZITE BY WHOLE PATTERN DECONVOLUTION
}

\author{
S. MATTHIES ${ }^{a, b}$, L. LUTTEROTTI $^{a, c}, K$ ULLEMEYER $^{d}$ \\ and H.-R. WENK ${ }^{\mathrm{a}, *}$ \\ ${ }^{a}$ Department of Geology and Geophysics, University of California, Berkeley, \\ CA 94720, USA; ${ }^{\text {b }}$ GKSS, D-21502 Geesthacht, Germany; ${ }^{\mathrm{c}}$ Dipartimento di \\ Ingegneria dei Materiali, Universita' di Trento, 38050 Mesiano (TN), Italy; \\ ${ }^{d}$ Frank Laboratory of Neutron Physics, JINR, Dubna, Russia
}

The recently developed RITA concept combines algorithms of the Rietveld structure analysis with those from modern texture analysis in order to get texture information from $d$-spacing diffraction spectra. It is demonstrated for a low symmetry material (quartz) and a minimum pole sphere covering by the measured spectra. The quality of the results underlines the efficiency of this new approach especially for time-of-flight neutron diffraction studies where access to the beam is limited. New interesting aspects for crystal structure refinement are discussed.

Keywords: Time-of-flight method; Rietveld analysis; Texture factor; Whole pattern fitting; Quartz

\section{INTRODUCTION}

The determination of the orientation distribution (OD) of textured polycrystals has largely relied on the analysis of pole figures that were measured with diffraction techniques. Various methods exist to determine the OD from a small number of complete or incomplete pole figures. This is efficient if diffraction peaks are well separated, as is the case, e.g. in cubic metals. Many materials, particularly those of low crystal symmetry and composites, have complex diffraction spectra

\footnotetext{
${ }^{*}$ Corresponding author.
} 
with many closely spaced and overlapping diffraction peaks. In these cases a different approach is more adequate.

Rietveld $(1967 ; 1969)$ introduced a method to determine the crystal structure from a continuous diffraction spectrum of a powder sample. His method relies on the premise that a diffraction spectrum is given by the incident beam characteristics, line-broadening/instrumental parameters (such as peak shape, background function, Lorentz-polarization corrections etc.), and structural parameters (cell parameters, atomic positions, temperature factors etc.). From the measured spectrum these parameters can be refined using a non-linear least squares method. Analytical functions, like the March-Dollase correction (Dollase, 1986), were incorporated to take into account intensity deviations due to preferred orientations. More recently this method has been expanded to include elements of modern texture analysis of polycrystalline samples, using the orientation distribution function (ODF) (Popa, 1992; Ferrari and Lutterotti, 1994; Lutterotti et al., 1997; Matthies et al., 1997; Von Dreele, 1997). With examples analysed so far, it could be shown that it is possible to determine (or to refine) simultaneously (or step by step) crystal structure, texture, microstructural parameters and residual stresses from a series of diffraction spectra, measured in different sample orientations.

In the conventional texture analysis many sample directions are measured on a few pole figures. With the new approach, a large number of diffraction peaks are considered, using possibly diffraction spectra for a few sample directions only. The OD resolution of both methods will be similar for an equivalent amount of experimental texture information (Matthies et al., 1997), but the "Rietveld Texture Analysis" (RITA) is particularly efficient for complex spectra and TOF neutron diffraction where the whole spectrum is recorded anyway.

\section{EXPERIMENTAL}

In this report we apply RITA to a sample of metamorphic quartzite. The quartzite is a clast from a metaconglomerate in Wildrose Canyon (Death Valley National Park, California). The texture has been determined previously by optical measurements of individual $c$-axes (L. Weiss, unpublished) and with conventional neutron diffraction 
methods (Helming et al., 1994). The $c$-axes form a crossed girdle as is typical of quartzites deformed at relatively low metamorphic grade (Sander, 1950).

A cylindrical sample, $2 \mathrm{~cm}$ in diameter and $2 \mathrm{~cm}$ long was measured at the neutron source of the JINR-Dubna (pulsed reactor IBR-2), using the technique described in detail by Walther et al. (1995). The $100 \mathrm{~m}$ TOF-line has a flux of $10^{6}$ neutrons $/\left(\mathrm{s} \mathrm{cm}^{2}\right)$ at the end of the neutron guide, and 7 detectors with an angular distance of $7.2^{\circ}$. Two arrangements of the detectors and a rotation of the sample around one axis (by $7.2^{\circ}$ steps) lead to a complete coverage of the whole pole sphere. Each spectrum contains about 2900 time channels. Spectra were recorded for the regular $7.2^{\circ}$ grid (650 sample directions) counting for $15 \mathrm{~min}$ at each sample position.

The arrangement of the detector banks at different $2 \Theta$ positions in the present experiment requires some non-trivial corrections. Different detectors see the same diffraction peak at different neutron wavelengths $\lambda$. Hence, the spectra must be normalized with respect to the energy distribution of the incoming neutrons, and the wavelength dependence of the scattering behavior must be considered ( $\lambda^{3}$ correction). Additionally, the non-equivalent detector positions require the consideration of the Lorentz effect $(1 / \sin \Theta \sin 2 \Theta)$. All these correction procedures, including the detector sensitivity, had to be included in the RITA algorithm for the present project. The new Dubna TOF spectrometer with all detectors at the same Bragg angle $\Theta$ will remarkably simplify the application of RITA in the future.

\section{RITA}

The RITA analysis follows the procedure characterized above and is described in detail by Matthies et al. (1997). Experimental information was available in the $d$-range $0.5-3.5 \AA$ containing 527 diffraction peaks. Though the technical run of the RITA program package was successfully tested for the whole amount of the experimental data, a reduced variant was chosen especially to demonstrate the characteristic power of the RITA concept that is able to work with a high degree of incompleteness of pole figures. Of the 650 measured spectra less than half (301) were used. The corresponding sample directions cover the central 
portion of the pole sphere up to a polar angle of about $40^{\circ}$ only. Of the 527 reflections only the (37) peaks having an intensity greater than $10 \%$ of the most intense reflection were used for the texture related calculations. One trial with 62 reflections ( $5 \%$ threshold) did not show remarkable improvements of the resulting texture data. Typical TOF spectra for different sample directions are shown in Fig. 1. The changing peak intensities are due to texture effects.

The crystal structure was assumed to be that of $\alpha$-quartz as reported in Table I; this structure has been kept fixed initially and refined only in the final stage. The $\alpha$-quartz has a trigonal crystal symmetry (32). This causes two complications: First, there are non-equivalent diffraction peaks such as 101 and 011 with identical $d$-spacing but different intensities. However, these strictly overlapped peaks can properly be resolved for texture related questions, using intensity weights given by the crystal

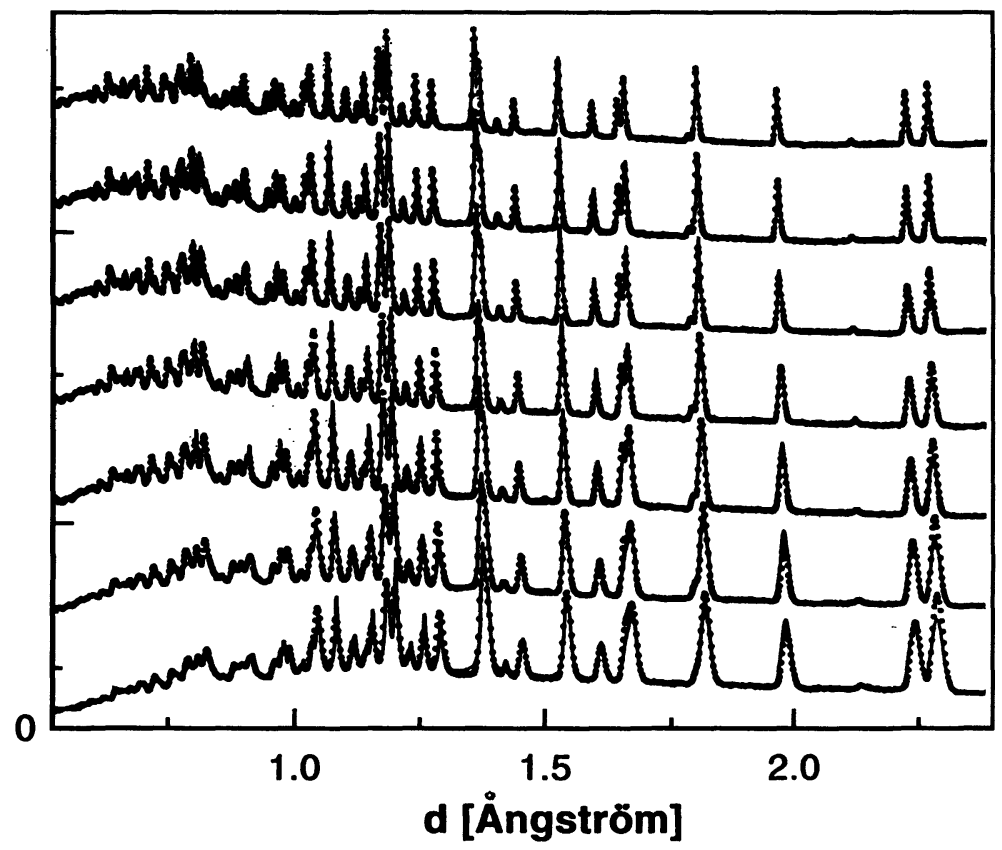

FIGURE 1 Typical TOF spectra of the quartzite sample for different sample directions (detectors). The solid lines give an impression on the fit quality using texture factors. 
TABLE I Values of the starting and refined crystal structure for $\alpha$-quartz (space group $\mathrm{P}_{2} 21$ ) using RITA. Values in parentheses are the estimated standard deviations as evaluated by the least squares algorithm

\begin{tabular}{lcc}
\hline & Starting structure $($ Wyckoff, 1982) & Refined \\
\hline$a(\AA)$ & 4.91304 & $4.91393(5)$ \\
$c(\AA)$ & 5.40463 & $5.40394(9)$ \\
$\mathrm{Si}(x, x, 0)$ & & \\
$x$ & 0.465 & $0.46323(7)$ \\
$B\left(\AA^{2}\right)$ & 0 & $0.4(2)$ \\
$\mathrm{O}(x, y, z)$ & & \\
$x$ & 0.415 & $0.41047(5)$ \\
$y$ & 0.272 & $0.26474(5)$ \\
$z$ & 0.787 & $0.78471(3)$ \\
$B\left(\AA^{2}\right)$ & 0 & $0.7(1)$ \\
\hline
\end{tabular}

structure (Baker et al., 1969). Secondly, quartz is enantiomorphic, existing in a right- and left-handed form. Due to the centrosymmetry of normal diffraction effects, this cannot be resolved from the available data by any method. Therefore commonly it is assumed that both forms have the same frequency and orientation distribution. For texture related problems effectively a center of symmetry is added and the resulting crystal symmetry (Laue group) considered is $\overline{3} 2 / \mathrm{m}$, the same point group as for calcite.

In a first step of the RITA scheme some instrumental parameters were refined. In particular the incident intensity, some background parameters, the instrumental resolution function and $d$-spacing related errors - one set for each detector, apart from the intensity as a unique parameter. For these refinements the known quartz structure and an isotropic "powder" spectrum have been used. This spectrum with an assumed random OD can be appproximated by a weighted mean of all spectra for the different sample directions. After these preliminary refinements the texture is determined using an iterative procedure as follows.

Applying a modification of the Le Bail algorithm from the conventional Rietveld analysis originally used for structure factor extraction in $a b$ initio crystal structure determinations (Le Bail et al., 1988), the intensities can be determined for all reflections of the sample direction dependent spectra. Because the structure factors are known by the fixed crystal structure, so-called texture factors can be determined for all reflections correspondingly. These texture factors, which are (initially as 
a rule quite ambiguously) related to the corresponding pole figure values, serve then as INPUT data for the direct WIMV algorithm (Matthies and Vinel, 1982) in order to get a first ODF-approximation. From this ODF "recalculated" pole figures can be determined (compatible in the sense of projections of one common OD) and will then be used as starting approximation of higher quality for the next Le Bail step a.s.o.

As in previous examples, this iterative process converges quite rapidly, i.e. the WIMV procedure leads always to better RP values (Matthies et al., 1988) characterizing the deviations between the "experimental" (in our case the Rietveld texture factors) and OD recalculated pole figures. The inspection of the RP values can indicate a low texture sensitivity or poor compatibility between values for different reflections $h k l$, and these can then be omitted from the next iteration steps. After few of these cycles (generally 2 or 3 are sufficient), also the crystal structure and microstructure parameters were refined together with the others.

The results for the quartzite sample are illustrated by some selected "experimental" pole figures (Fig. 2), by pole figures recalculated from the ODF (Fig. 3), and by the ODF of the last WIMV step after convergence (Fig. 4). The ODF is displayed by $\sigma$-sections that show minimal distortions of component forms due to the complicated orientation space metrics (Matthies et al., 1990). Three iteration steps were sufficient in order to get RP values in the order of $5 \%$. A comparison of Figs. 2 and 3 gives a visual impression on the fit quality. Due to the statistics of the experimental data and the transformation of the $7.2^{\circ}$-grid data into the $5^{\circ}$-working grid of WIMV, the resulting OD of Fig. 4 is rather rugged. Therefore a Gaussian OD filtering procedure with a width $b=7.5^{\circ}$ (Matthies and Vinel, 1994) was applied to this 'noisy' ODF preserving its texture index and order of RP values. The corresponding results shown in Figs. 5 and 6 are more comprehensible. For representations and post-processing some programs contained in the BEARTEX package were used (Wenk et al., 1998).

A specific property of the quartzite sample is its relatively high phon of 0.4 . It means that about $40 \%$ of the crystallites of the sample are randomly oriented. In such cases an additional correction procedure of "remaining ghosts" in accordance with the WIMV concept is recommended and was already performed for the ODF shown in Fig. 4. 

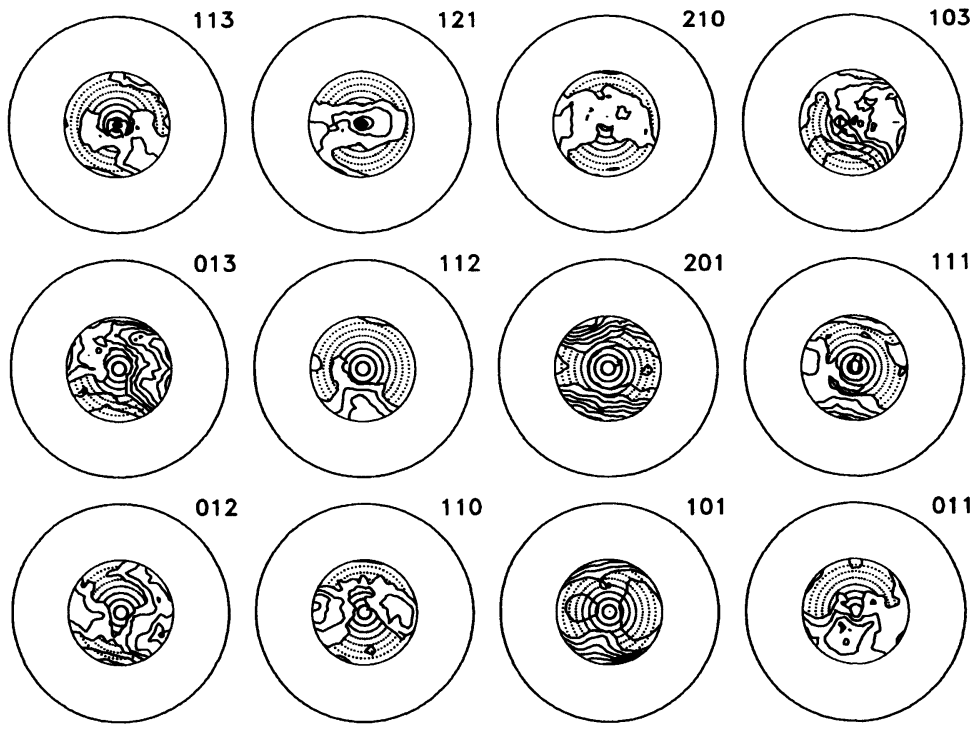

FIGURE 2 Some of the used incomplete "experimental" pole figures constructed by texture factors from the Le Bail step - third iteration cycle. Equal area projection. Isolines: $0.6,0.8 ., 1.0,1.1,(0.1)$ 1.6. Dotted area below 1 .
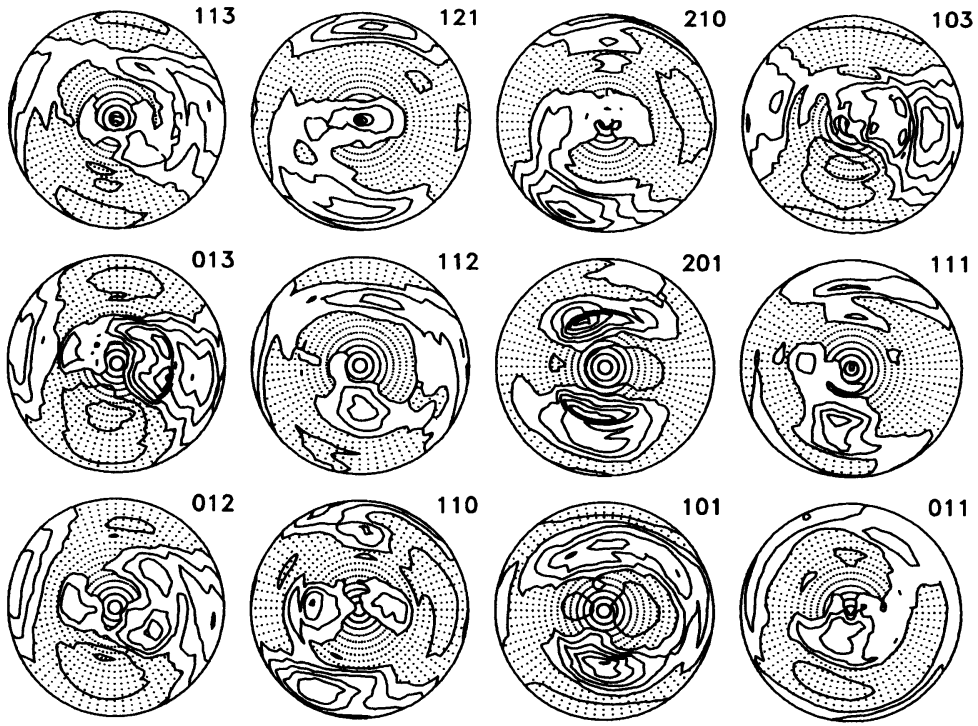

FIGURE 3 Recalculated pole figures from the WIMV-ODF (cf. Fig. 4) - third iteration cycle. Representation like in Fig. 2. 


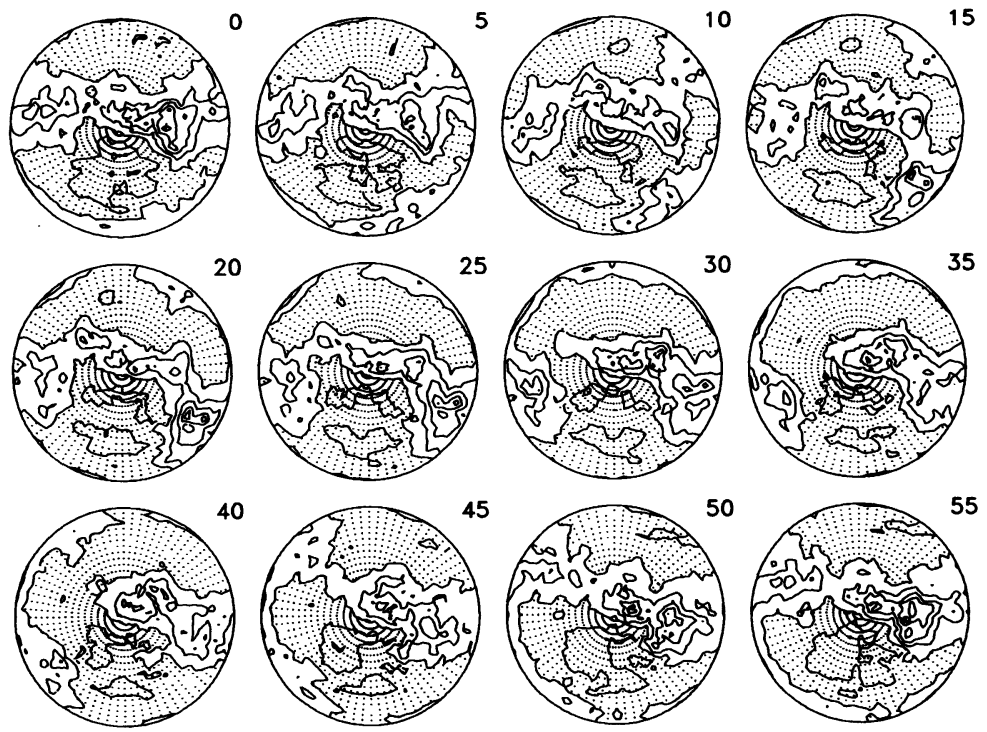

FIGURE 4 The required ODF - third iteration cycle. Sigma sections. Azimuth $\alpha$, polar angle $\beta$ of the $g=\{\alpha, \beta, \gamma\}$ variant of Euler angles; $\sigma=(\alpha+\gamma) / 2$. Equal area projection. Isolines: $0.5,(0.5) 3.5$. Dotted area below 1 .
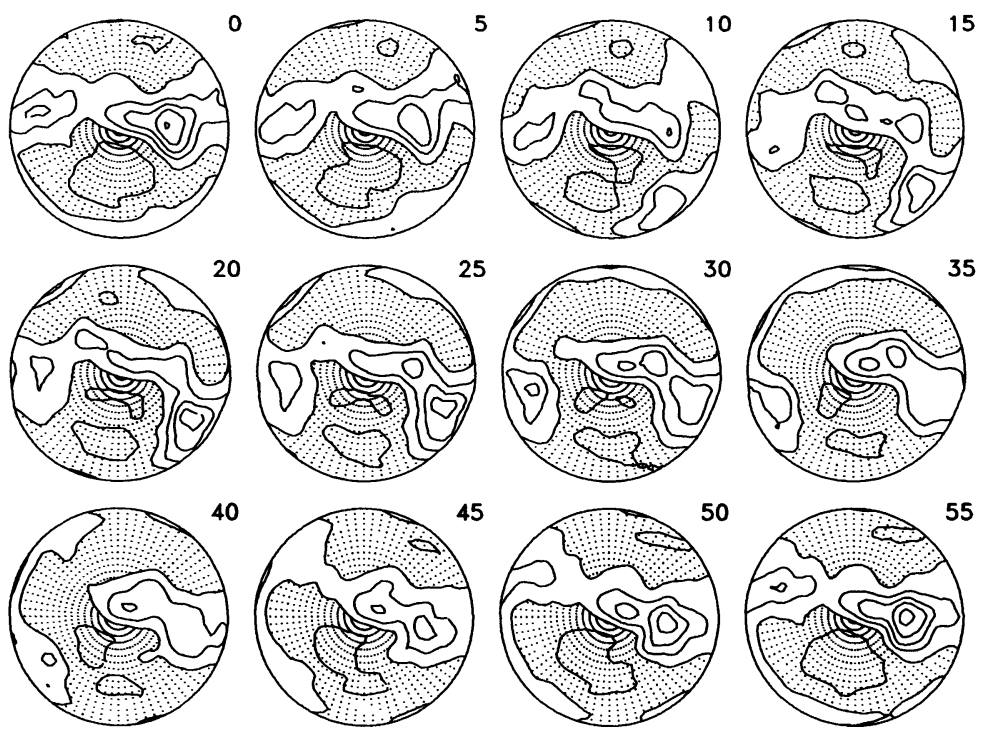

FIGURE 5 The $7.5^{\circ}$-filtered ODF from Fig. 4. Same parameters of representation. 

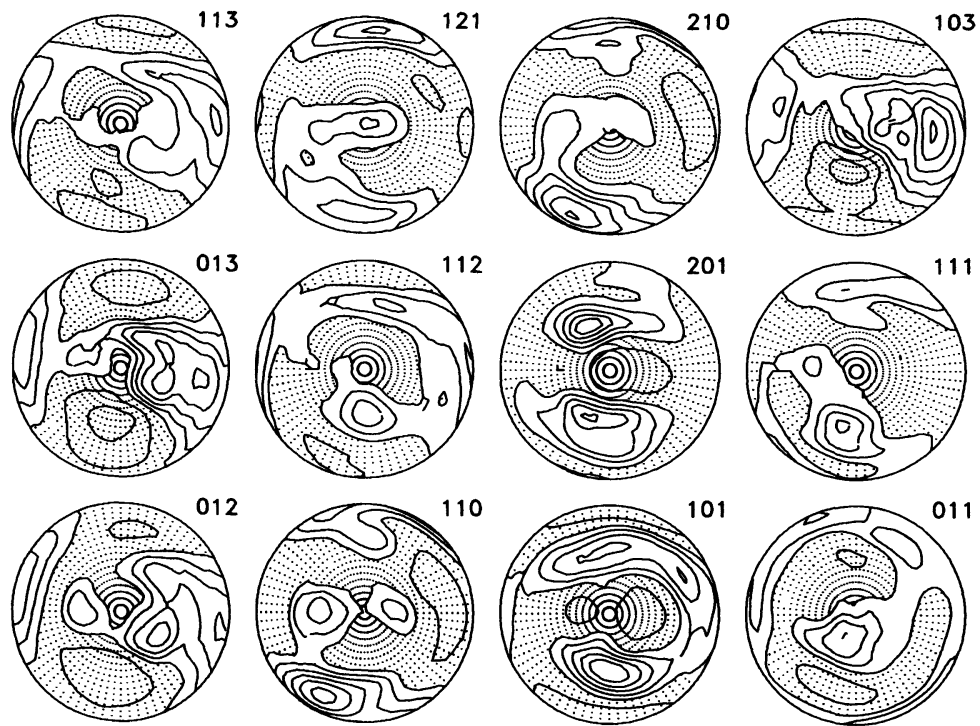

FIGURE 6 Recalculated pole figures using the ODF of Fig. 5 (cf. Fig. 3).

From a given OD any pole figures can be calculated. Of particular interest is the $c$-axis pole figure (Fig. 7(a)) that can be interpreted as the "sum" of all $\sigma$-sections of the ODF shown in Fig. 5. It compares reasonably with that obtained by the conventional method of direct pole figure measurements (Fig. 7(b), Helming et al., 1994) with a broad and asymmetric girdle. The concentration of some $c$-axes near the periphery in the Dubna data is not present in the conventional analysis and in the optical grain by grain measurements. The difference could have several reasons like the greater amount of experimental data in the Dubna TOF measurements, problems with the background correction in the conventional analysis, relatively poor statistics in the optical experiment, or possibly systematic aberrations in the Dubna spectra.

Concerning the crystal structure refinement it should be mentioned that it is possible to obtain very accurate results also in the case of a textured sample, as it is evident from Table I. The large amount of data is responsible for the very low estimated standard deviations obtained. Some indicators of the correctness of the method are given by the reasonable Debye-Waller factor values (the $B$ 's in Table I), that normally 
(a)

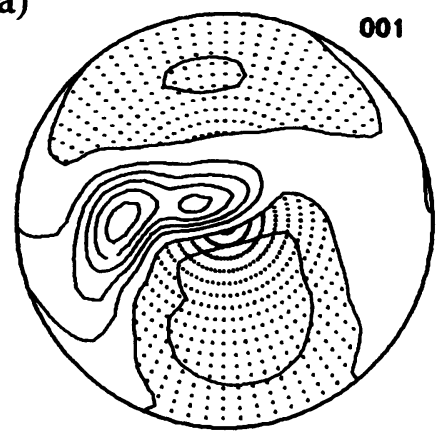

(b)

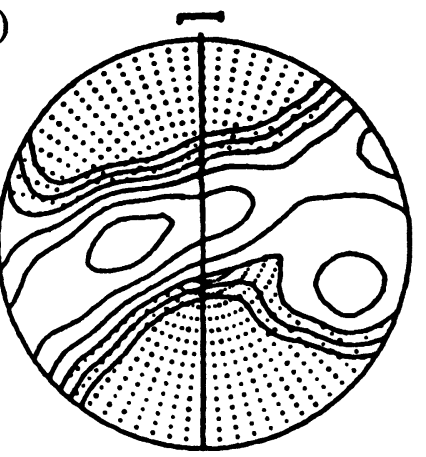

FIGURE 7 (a) The 001 pole figure from the OD of Fig. 5 (RITA). (b) The 001 pole figure from a conventional analysis (Helming et al., 1994).

are considered as not sufficiently reliable from diffraction experiments. The employment of neutron radiation has also enhanced the accuracy of the determination of the oxygen position. That is the present methodology demonstrates the possibility not only of a better refinement of known structures but also for the determination of partially unknown or incompletely refined substances.

A real textured sample can be considered as lying in between an ideal powder sample with a random $O D$ and a single crystal. Using the demonstrated procedure in combination with ab initio methodologies it would be possible to solve complicated structures for which single crystals are not available. In such cases commonly the powder methods fail due to the impossibility to separate heavily overlapping reflections or real coincidences. A textured sample with sample direction dependent spectra can in principle provide sufficient information in order to discriminate such reflections for a successful solution.

\section{CONCLUSIONS}

It was demonstrated that the RITA concept can also be used to determine textures of low symmetry materials such as quartz from TOF diffraction spectra. The analysis can be carried out with only a few measured spectra, making it efficient for neutron diffraction studies where access to the beam is limited. The agreement between measured 
and recalculated pole figures assesses the quality of the experimental data and of the OD determination. RITA opens some new perspectives for crystal structure refinement.

\section{Acknowledgements}

We are appreciative for support of the RITA project through NSF grant EAR 94-17580, a grant from IGPP Los Alamos and the Center of Materials Science at Los Alamos (through D. Parkin). Access to the Dubna texture goniometer is gratefully acknowledged.

\section{References}

Baker, D.W., Wenk, H.R. and Christie, J.M. (1969). J. Geol. 77, 144-172.

Dollase, W.A. (1986). J. Appl. Cryst. 19, 267-272.

Ferrari, M. and Lutterotti, L. (1994). J. Appl. Phys. 76, 7246-7255.

Helming, K., Wenk, H.R., Choi, C.S. and Schaefer, W. (1994). In Textures of Geological Materials, H.J. Bunge, S. Siegesmund, W. Skrotzki and K. Weber (Eds.), DMG. p. 303.

Le Bail, A., Durroy, H. and Fourquet, J.L. (1988). Mater. Res. Bull. 23, 447-452.

Lutterotti, L., Matthies, S. and Wenk, H.R. (1997). J. Appl. Phys. 81, 594-600.

Matthies, S. and Vinel, G.W. (1982). Phys. Status Solidi B 112, 111-120.

Matthies, S., Wenk, H.R. and Vinel, G.V. (1988). J. Appl. Cryst. 21, 285-304.

Matthies, S., Helming, K. and Kunze, K. (1990). Phys. Status Solidi B 157, 71-83; 489-507.

Matthies, S. and Vinel, G.W. (1994). Mater. Sci. Forum 157-162, 1641-1646.

Matthies, S., Lutterotti, L. and Wenk, H.R. (1997). J. Appl. Cryst. 30, 31-42.

Popa, N.C. (1992). J. Appl. Cryst. 25, 611-616.

Rietveld, H.M. (1967). Acta Cryst. 22, 151.

Rietveld, H.M. (1969). J. Appl. Cryst. 2, 65-72.

Sander, B. (1950). Einführung in die Gefügekunde der Geologischen Körper. Springer Verlag, Vienna.

Von Dreele, R. (1997). J. Appl. Cryst. 30, 517-525.

Walter, K., Heinitz, J., Ullemeyer, K., Betzl, M. and Wenk, H.R. (1995). J. Appl. Cryst. 28, 503-507.

Wenk, H.-R., Matthies, S., Donovan, J. and Chateigner, D. (1998). J. Appl. Cryst. (in press).

Wyckoff, R.W.G. (1982). Crystal Structures. 2nd Ed., Vol. 1. R.E. Krieger Pub., Florida. 